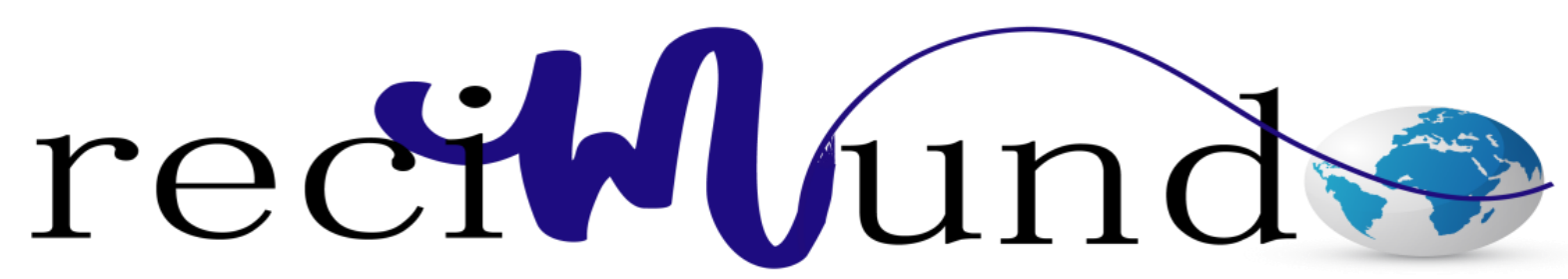

Revista Cientifica Mundo de la Investigación y el Conocimiento

Javier Fabricio Valdez Zambrano ${ }^{\text {a; }}$ A Alex William Marín Cherrez ${ }^{\text {b; }}$ Verónica

Alexandra Muñoz Avilés ${ }^{c}$; Joselyn Anabell Minda Reyes ${ }^{d}$

Atención prehospitalaria en trauma craneoencefálico

Prehospital care in craniocerebral trauma

Revista Científica Mundo de la Investigación y el Conocimiento. Vol. 3 núm.3, septiembre, ISSN: 2588-073X, 2019, pp. 183-200

DOI: $10.26820 /$ recimundo/3.(3).septiembre.2019.183-200

URL: http://recimundo.com/index.php/es/article/view/517

Código UNESCO: 3205 Medicina Interna

Tipo de Investigación: Artículo de Revisión

Editorial Saberes del Conocimiento

Recibido: 15/05/2019

Aceptado: 23/06/2019

Publicado: 30/09/2019

Correspondencia: $\underline{\text { fa_vaza@ hotmail.com }}$

a. Médico; Investigador Independiente; Guayaquil, Ecuador; fa_vaza@ hotmail.com

b. Médico; Investigador Independiente; Guayaquil, Ecuador; alexmuchinch@ gmail.com

c. Médico; Investigador Independiente; Guayaquil, Ecuador; verito456@yahoo.com

d. Médico; Investigador Independiente; Guayaquil, Ecuador; josyanabell@ hotmail.com 


\section{Atención prehospitalaria en trauma craneoencefálico}

Vol. 3, núm. 3., (2019)

Javier Fabricio Valdez Zambrano; Alex William Marín Cherrez; Verónica Alexandra Muñoz Avilés; Joselyn Anabell Minda Reyes

\section{RESUMEN}

La mayor parte de los malos resultados en pacientes con lesión cerebral traumática grave están relacionados con la presencia de una alta incidencia de falla en el manejo prehospitalario. Por lo tanto, el conocimiento de estas variables y manejo oportuno de la enfermedad en el período prehospital puede mejorar significativamente los resultados y disminuir la mortalidad. La pauta Brain Trauma Foundation sobre "manejo prehospitalario", publicado en 2008 podría proporcionar los protocolos estandarizados para el manejo de pacientes con lesión cerebral traumática. En base a la evidencia, mayoría de las estrategias de gestión forman parte de la rápida corrección de la hipoxemia e hipotensión, los dos más importantes predictores de mortalidad. Sin embargo, todavía hay una necesidad de definir los objetivos para el tratamiento de la hipotensión y la inclusión de nuevos tratamientos de las vías respiratorias, así como dispositivos de control adecuados para garantizar una mejor gestión de la intubación y la ventilación. La solución salina isotónica debe utilizarse como la primera opción para la reposición de líquidos. La hipotermia prehospitalaria tiene más efectos adversos; Por lo tanto, esto debe evitarse.

Palabras Claves: Pruebas; Manejo prehospitalario; Lesión cerebral secundaria; Daño cerebral. 


\title{
Atención prehospitalaria en trauma craneoencefálico
}

Vol. 3, núm. 3., (2019)

Javier Fabricio Valdez Zambrano; Alex William Marín Cherrez; Verónica Alexandra Muñoz

Avilés; Joselyn Anabell Minda Reyes

\begin{abstract}
Most of the poor results in patients with severe traumatic brain injury are related to the presence of a high incidence of prehospital management failure. Therefore, knowledge of these variables and timely management of the disease in the pre-hospital period can significantly improve the results and decrease mortality. The Brain Trauma Foundation guideline on "prehospital management," published in 2008, could provide standardized protocols for the management of patients with traumatic brain injury. Based on the evidence, most management strategies are part of the rapid correction of hypoxemia and hypotension, the two most important predictors of mortality. However, there is still a need to define the objectives for the treatment of hypotension and the inclusion of new airway treatments, as well as adequate control devices to ensure better management of intubation and ventilation. Isotonic saline should be used as the first option for fluid replacement. Prehospital hypothermia has more adverse effects; Therefore, this should be avoided.
\end{abstract}

Key Words: Tests; Prehospital management; Secondary brain injury; Brain damage. 


\section{Atención prehospitalaria en trauma craneoencefálico}

Vol. 3, núm. 3., (2019)

Javier Fabricio Valdez Zambrano; Alex William Marín Cherrez; Verónica Alexandra Muñoz Avilés; Joselyn Anabell Minda Reyes

\section{Introducción.}

La lesión cerebral traumática (LCT) es una importante carga en el sistema de atención de salud en todo el mundo.Por otra parte, de los pacientes que sobrevivieron a la lesión primaria, algunos pueden permanecer con discapacidad a largo plazo. La mayor parte de estos resultados se relacionan con la presencia de una alta incidencia de problemas prehospitalarios cerebrales secundarios. Por lo tanto, el conocimiento de estas variables y manejo oportuno de la enfermedad en el período prehospital puede mejorar significativamente los resultados y disminuir la mortalidad(Wu, y otros, 2008)

La práctica clínica "manejo prehospitalario” por las directrices Brain Trauma Foundation (BTF) basada en la evidencia ha sido modificada y revisada en 2008.Estas directrices podrían proporcionar los protocolos estandarizados para el manejo de pacientes con lesión cerebral traumática, a partir de entonces, los resultados de diversos ensayos han sido publicados y el foco principal de estos es principalmente para minimizar la lesión cerebral secundaria después de los auxilios primarios. Sin embargo, los resultados y conclusiones de estos ensayos están todavía a ser adoptadas por las directrices estándar.

En este artículo, hemos resumido los diversos aspectos de la atención prehospitalaria, así como el manejo inicial en servicio de urgencias en pacientes con lesión cerebral traumática, incluyendo la evidencia emergente sobre todo después del BTF 2008.(Badjatia, y otros, 2008) 


\section{Atención prehospitalaria en trauma craneoencefálico}

Vol. 3, núm. 3., (2019)

Javier Fabricio Valdez Zambrano; Alex William Marín Cherrez; Verónica Alexandra Muñoz Avilés; Joselyn Anabell Minda Reyes

Métodos.

Para el desarrollo de este proceso investigativo, se plantea como metodología la encaminada hacia una orientación científica particular que se encuentra determinada por la necesidad de indagar en forma precisa y coherente una situación, en tal sentido (Davila, 2015) define la metodología "como aquellos pasos previos que son seleccionados por el investigador para lograr resultados favorables que le ayuden a plantear nuevas ideas".(p.66)

Lo citado por el autor, lleva a entender que el desarrollo de la acción investigativa busca simplemente coordinar acciones enmarcadas en una revisión bibliográfica con el fin de complementar ideas previas relacionadas a la atención prehospitalaria en traumas craneoencefálicos a través de una revisión de literatura, para así finalmente elaborar un cuerpo de consideraciones generales que ayuden a ampliar el interés propuesto.

\section{Tipo de Investigación.}

Dentro de toda práctica investigativa, se precisan acciones de carácter metodológico mediante las cuales, se logra conocer y proyectar los eventos posibles que la determinan, así como las características que hacen del acto científico un proceso interactivo ajustado a una realidad posible de ser interpretada. En este sentido, se puede decir, que la presente investigación corresponde al tipo documental, definido por Castro (2016), "se ocupa del estudio de problemas planteados a nivel teórico, la información requerida para abordarlos se encuentra básicamente en materiales impresos, audiovisuales y /o electrónicos”. (p.41). 


\section{Atención prehospitalaria en trauma craneoencefálico}

Vol. 3, núm. 3., (2019)

Javier Fabricio Valdez Zambrano; Alex William Marín Cherrez; Verónica Alexandra Muñoz Avilés; Joselyn Anabell Minda Reyes

En consideración a esta definición, la orientación metodológica permitió la oportunidad de cumplir con una serie de actividades inherentes a la revisión y lectura de diversos documentos donde se encontraron ideas explicitas relacionadas con los tópicos encargados de identificar a cada característica insertada en el estudio. Por lo tanto, se realizaron continuas interpretaciones con el claro propósito de revisar aquellas apreciaciones o investigaciones propuestas por diferentes investigadores relacionadas con el tema de interés, para luego dar la respectiva argumentación a los planteamientos, en función a las necesidades encontradas en la indagación.

\section{Fuentes Documentales.}

El análisis correspondiente a las características que predomina en el tema seleccionado, llevan a incluir diferentes fuentes documentales encargadas de darle el respectivo apoyo y en ese sentido cumplir con la valoración de los hechos a fin de generar nuevos criterios que sirven de referencia a otros procesos investigativos. Para (CASTRO, 2016) las fuentes documentales incorporadas en la investigación documental o bibliográfica, "representa la suma de materiales sistemáticos que son revisados en forma rigurosa y profunda para llegar a un análisis del fenómeno".(p.41). Por lo tanto, se procedió a cumplir con la realización de una lectura previa determinada para encontrar aquellos aspectos estrechamente vinculados con el tema, con el fin de explicar mediante un desarrollo las respectivas apreciaciones generales de importancia.

\section{Técnicas para la Recolección de la Información.}

La conducción de la investigación para ser realizada en función a las particularidades que determinan a los estudios documentales, tiene como fin el desarrollo de un conjunto de acciones encargadas de llevar a la selección de técnicas estrechamente vinculadas con las características del 


\section{Atención prehospitalaria en trauma craneoencefálico}

Vol. 3, núm. 3., (2019)

Javier Fabricio Valdez Zambrano; Alex William Marín Cherrez; Verónica Alexandra Muñoz Avilés; Joselyn Anabell Minda Reyes

estudio. En tal sentido, (Bolívar, 2015), refiere, que es "una técnica particular para aportar ayuda a los procedimientos de selección de las ideas primarias y secundarias". (p. 71).

Por ello, se procedió a la utilización del subrayado, resúmenes, fichaje, como parte básica para la revisión y selección de los documentos que presentan el contenido teórico. Es decir, que mediante la aplicación de estas técnicas se pudo llegar a recoger informaciones en cuanto a la revisión bibliográfica de los diversos elementos encargados de orientar el proceso de investigación. Tal como lo expresa, (Bolívar, 2015) "las técnicas documentales proporcionan las herramientas esenciales y determinantes para responder a los objetivos formulados y llegar a resultados efectivos" (p. 58). Es decir, para responder con eficiencia a las necesidades investigativas, se introdujeron como técnica de recolección el método inductivo, que hizo posible llevar a cabo una valoración de los hechos de forma particular para llegar a la explicación desde una visión general.

Asimismo, se emplearon las técnicas de análisis de información para la realización de la investigación que fue ejecutada bajo la dinámica de aplicar diversos elementos encargados de determinar el camino a recorrer por el estudio, según, (Bolívar, 2015) las técnicas de procesamiento de datos en los estudios documentales "son las encargadas de ofrecer al investigador la visión o pasos que debe cumplir durante su ejercicio, cada una de ellas debe estar en correspondencia con el nivel a emplear" (p. 123). Esto indica, que para llevar a cabo el procesamiento de los datos obtenidos una vez aplicado las técnicas seleccionadas, tales como: fichas de resumen, textual, registros descriptivos entre otros, los mismos se deben ajustar al nivel que ha sido seleccionado. 


\section{Atención prehospitalaria en trauma craneoencefálico}

Vol. 3, núm. 3., (2019)

Javier Fabricio Valdez Zambrano; Alex William Marín Cherrez; Verónica Alexandra Muñoz Avilés; Joselyn Anabell Minda Reyes

\section{Resultados.}

\section{Etiopatogenia.}

La causa más común de incitación a una lesión en la cabeza es mediante accidente de vehículo automotor. Las otras causas de lesión cerebral traumática son caídasdesde alturas, agresiones físicas, accidentes en el hogar, oficinas omientras se practica deportes, disparos y heridas por explosión. La lesión cranealpuede ser una lesión cerrada (contundente) o abierta (penetrante) yse clasifica además en lesión primaria y lesión secundaria.Esta clasificación es bastante más útil si se considera las estrategias terapéuticas y preventivas [Tabla 1].

El tejido cerebral destruido por el impacto primario es muy difícilpara ser rescatado de una lesión cerebral secundaria, la secuela de la lesión primaria, es potencialmente tratable y la mayoría de las terapias sondirigidas a prevenir estas secuelas. Esta lesión secundariase amplifica principalmente por varios cofactores, incluidoshipoxemia, hipotensión, hipercarbia, hipoglucemia,hiperglucemia, hipotermia / hipertermia, convulsiones,etc. La morbilidad, mortalidad y discapacidad a largo plazodepende sustancialmente de la gestiónde la lesión cerebral secundario. Por lo tanto, resultado funcionalpuede mejorarse con una buena atención médica intensiva y conintervenciones quirúrgicas rápidas. (Pearson, Ovalle, Faul, Sasser, \& SM., 2012)

Hiperglucemia, la hipotermia / hipertermia, convulsiones, etc. La morbilidad, la mortalidad y discapacidad a largo plazo dependen sustancialmente de la gestión de insulto cerebral secundario. Por lo tanto, el resultado funcional puede ser mejorada mediante una buena atención médica intensiva y por las intervenciones quirúrgicas inmediatas.(Myburgh, y otros, 2008) 


\section{Atención prehospitalaria en trauma craneoencefálico}

Vol. 3, núm. 3., (2019)

Javier Fabricio Valdez Zambrano; Alex William Marín Cherrez; Verónica Alexandra Muñoz Avilés; Joselyn Anabell Minda Reyes

\section{Evaluación.}

Parámetros fisiológicos incluyendo una escala de coma de Glasgow (GCS), la presión arterial sistólica y la frecuencia respiratoria son predictores útiles para el resultado y pueden ser utilizados en el triaje pre-hospitalaria de pacientes con LCT. La GCS es un sistema rápido, reproducible de puntuación que tiene aceptación en todo el mundo y se utiliza para definir la gravedad de LCT, con TCE grave definida como GCS de $\leq 8$. Se basa en la apertura ocular, respuesta verbal y la mejor respuesta motora. Uno de los primeros, no sedado GCS se ha demostrado para predecir el pronóstico. La puntuación total más baja indica un daño fatal probable, especialmente si ambas pupilas no responden a la luz y las respuestas oculovestibular están ausentes. Las puntuaciones iniciales más altas tienden a predecir mejor recuperación.Los datos de la (NTDB) Programa Nacional por Muestreo de 2007 Nacional de Trauma Data Bank mostraron que después de controlar la edad, el género, la raza, el Injury Severity Score (ISS) y la longitudde estancia en el hospital, los pacientes que tenían una puntuación GCS $\leq 13$ eran 17 veces más propensos a morir que los pacientes con TCE que tuvieron una mayor puntuación GCS(Pearson, Ovalle, Faul, Sasser, \& SM., 2012).

El otro sistema de puntuación, la simplificada puntuación motora (SMS), una medida de tres puntos de la gravedad de LCT, se encuentra también para llevar a cabo igualmente bien como GCS y se valida en muchos ensayos. En una revisión retrospectiva de 52,412 pacientes, la sensibilidad, la especificidad y el área bajo el receptor - curvas características de funcionamiento fueron similares entre el SMS y GCS para todos los resultados.La sensibilidad para la mortalidad fue del 72,2\% para los SMS y el 74,6\% de GCS. La sensibilidad para la lesión cerebral traumática 


\section{Atención prehospitalaria en trauma craneoencefálico}

Vol. 3, núm. 3., (2019)

Javier Fabricio Valdez Zambrano; Alex William Marín Cherrez; Verónica Alexandra Muñoz Avilés; Joselyn Anabell Minda Reyes

fue 40,8\% para los SMS y el 45,4\% de GCS. La sensibilidad para la intervención neuroquirúrgica fue del 52,9\% para SMS y 60,0\% para GCS(Caterino \& Raubenolt, 2012). Sin embargo, la gravedad y el pronóstico se predicen con más precisión considerando también la tomografía computarizada (TC) hallazgos de exploración y otros factores clínicos incluyendo un reflejo de la pupila perturbado e hipotensión arterial. Otras investigaciones, como los rayos X y ultrasonido también son útiles para descartar otras lesiones importantes.

Se requiere una TC temprana del cerebro sin contraste para guiar la terapia subsiguiente. De hecho, las instalaciones TC situado en el trauma o la sala de emergencias se ha encontrado para reducir el tiempo para adquirir imágenes de TC y mejora la mortalidad global. Una vez que se mantiene la vía aérea, la respiración o ventilación y la presión arterial, uno debe concentrarse en el manejo de la hipertensión intracraneal y evaluar las otras lesiones asociadas.

\section{Transporte.}

También se encontró que el resultado en pacientes con TCE a ser afectados por los métodos de transporte, la duración y el médico o paramédicos líderes del equipo. En algunos sistemas, los equipos médicos de aire pueden proporcionar un nivel superior de atención, pero pueden requerir mayor tiempo de transporte. Basándose en el análisis de datos (2049 pacientes) de dos ensayos aleatorios de reanimación prehospitalaria hipertónica, pacientes transportados por el aire (en comparación con el transporte de tierra) tenían tasas más altas de intubación pre-hospitalaria (81\% vs. 36\%), recibió fluidos más intravenosas (media 1,3 L vs. 0,8 L) y tuvieron lapsos de tiempoprehospitalarios más largos (media 76,1 min vs. 43,5 min)(Bulger, y otros, 2012). Sin embargo, no hubo un impacto significativo del medio de transporte en los resultados de 


\section{Atención prehospitalaria en trauma craneoencefálico}

Vol. 3, núm. 3., (2019)

Javier Fabricio Valdez Zambrano; Alex William Marín Cherrez; Verónica Alexandra Muñoz Avilés; Joselyn Anabell Minda Reyes

supervivencia. Del mismo modo, en un estudio retrospectivo de 194 pacientes, los servicios médicos de emergencia a base de aire se asociaron con una menor tasa de mortalidad (21\% vs. 25\%), así como discapacidades neurológicas (25\% vs. $31 \%$ )(Berlot, y otros, 2009). Por lo tanto, existe la necesidad de integrar el sistema de transporte más eficaz a través del cual los pacientes de LCT pueden desplazarse a un centro de trauma especializada dentro del mínimotiempo posible. El tiempo es un factor crucial para la aparición, así como la prevención de la lesión cerebral secundaria; Por lo tanto, el vehículo de transporte debe tener todos los posibles dispositivos de drogas / equipos y de supervisión,los personales médicos de emergencia que acompañan también deben tener un profundo conocimiento de importantes factores de riesgo y estrategias de gestión basadas en las guías para asegurar la prevención sustancial de daño cerebral secundario.

\section{El tratamiento inicial.}

Las directrices de soporte vital en trauma (ATLS) proporcionan un protocolo estandarizado para acercarse al paciente gravemente herido, con especial atención a las vías respiratorias, la respiración y la circulación.

\section{Hipoxemia.}

La primera consideración más importante en la LCT es el mantenimiento de una vía aérea clara y sin obstáculos. La hipoxemia es los factores más importantes relacionados con un peor resultado y por lo tanto requiere una atención urgente.En un estudio prospectivo multicéntrico de 150 pacientes, se encontró que la mortalidad a $20 \%$ en pacientes sin lesiones secundarias; sin embargo, se incrementó a 37\% durante los episodios hipóxicos(Chi, y otros, 2006). Los pacientes 


\section{Atención prehospitalaria en trauma craneoencefálico}

Vol. 3, núm. 3., (2019)

Javier Fabricio Valdez Zambrano; Alex William Marín Cherrez; Verónica Alexandra Muñoz Avilés; Joselyn Anabell Minda Reyes

con TCE grave deben ser intubado por inducción de secuencia rápida por personal especializado.

La columna cervical debe ser protegida y siempre debe suponerse que está lesionado hasta definitivamente aclarado, mientras se asegura la vía aérea. Un ensayo controlado aleatorio mostró que intubación de secuencia rápida (RSI) realizado por paramédicos se asoció con un aumento en la tasa de resultado neurológico favorable a los 6 meses en comparación con la intubación en el hospital; esto pone de manifiesto la importancia del control de la vía aérea inmediata en casos de TCE grave(Bernard, y otros, 2010). Además, el uso de RSI con el uso apropiado de los agentes anestésicos es de suma importancia para prevenir eventos adversos relacionados con aspiracióny, simultáneamente, evitar sobretensiones simpático incitado por manipulaciones de las vías respiratorias; sin embargo, la evitación de la hipotensión debe garantizarse.

La técnica y el equipo también son igualmente importantes para gestionar las intubaciones prehospitalaria, especialmente por los paramédicos. En este sentido, el papel de videolaringoscopios y otros equipos de intubación difícil sin duda puede dar mejores posibilidades para la gestión de las vías respiratorias y así evitaría las lesiones cerebrales secundarias relacionadas con la hipoxemia.

\section{Hipercapnia / hipocarbia.}

Los pacientes intubados deben ser ventilados para mantener la normocapnia (PaCO2 35 y $40 \mathrm{mmHg}$ ). Se recomienda la monitorización de la saturación de oxígeno y la capnografía en pacientes con lesiones graves en la cabeza para evitar la hipoxemia no reconocida o los cambios en la ventilación [Tablas 3, 4]. [4] Esto se evalúa mejor por capnografía de marea final, y generalmente corresponde a EtCO2 30-35 mmHg. [4] En el análisis de datos de 11,000 pacientes, 


\section{Atención prehospitalaria en trauma craneoencefálico}

Vol. 3, núm. 3., (2019)

Javier Fabricio Valdez Zambrano; Alex William Marín Cherrez; Verónica Alexandra Muñoz Avilés; Joselyn Anabell Minda Reyes

tanto la hipocapnia como la hipercapnia se asociaron con peores resultados en pacientes intubados. [19] En otra revisión retrospectiva de pacientes, tanto la hipocarbia (PaCO2- $\square 35 \mathrm{mmHg}$ ) como la hipercarbia (PaCO2- $\square 45 \mathrm{mmHg}$ ) se asociaron con un aumento sustancial de la mortalidad hospitalaria (77\% y 61\%, respectivamente). [20] Un estudio sobre el manejo basado en pautas en la ventilación prehospitalaria ( $\mathrm{PaCO} 2 \square 29 \mathrm{mmHg}$ ) en pacientes (sin signos de hernia) mostró que la mortalidad hospitalaria fue del $29 \%$ entre aquellos en los que se alcanzaron los niveles de las pautas y 46\% (16 de 35 ) en aquellos en los que no se alcanzaron los niveles de referencia prehospitalarios. De manera similar, una revisión retrospectiva de 851 pacientes con ventilación dirigida ( $\mathrm{PaCO} 2$ 30-39 mmHg) en el departamento de emergencias también encontró una disminución de la mortalidad (21.2\% vs. 33.7\%) en comparación con la ventilación lograda fuera del rango objetivo. [21] Por lo tanto, debe garantizarse la normoventilación prehospitalaria. Sin embargo, en casos con signos de potencial hernia cerebral, la hiperventilación transitoria puede ser una opción. Hay pruebas sólidas de que la capnografía puede afectar el resultado. Por lo tanto, se recomienda la capnografía continua en todos los pacientes con LCT ventilados. [21,22] Como los valores de ETCO2 pueden confundirse por una baja perfusión tisular, la ventilación a largo plazo debe guiarse por el muestreo frecuente de gases en sangre. [23] La frecuencia del muestreo de gases en sangre aún no se ha cuantificado.

La oxigenación y la ventilación pueden volverse difíciles si hay otras lesiones importantes, particularmente lesiones en el pecho, aspiración de contenido gástrico o edema pulmonar agudo. Se debe utilizar la presión espiratoria final adecuada (PEEP) adecuada. No hay evidencia de que 


\section{Atención prehospitalaria en trauma craneoencefálico}

Vol. 3, núm. 3., (2019)

Javier Fabricio Valdez Zambrano; Alex William Marín Cherrez; Verónica Alexandra Muñoz Avilés; Joselyn Anabell Minda Reyes

la PEEP de 10 mmHg afecte el flujo sanguíneo cerebral o eleve la presión intracraneal (PIC) y, de hecho, la PIC puede mejorar con una mejor oxigenación. [24]

\section{Hipotensión / hipertensión.}

La hipotensión es una gran lesión cerebral secundaria y los estudios han demostrado que un solo episodio de hipotensión empeora dramáticamente el resultado. La hipotensión debe tratarse con reanimación con líquidos para la volemia y, si es necesario, se utilizan vasopresores para mantener una presión arterial óptima. De acuerdo con BTF, antes de la inserción de un control ICP, se recomienda un MAP $\geq 80 \mathrm{mmHg}$. La justificación de un MAP $\geq 80 \mathrm{mmHg}$ es mantener la presión de perfusión cerebral (CPP) $\geq 60 \mathrm{mmHg}$ para un umbral de tratamiento de ICP $\square 20$ mmHg(Bullock \& Povlishock, 2007). Después de la inserción de un monitoreo de ICP, la gestión de MAP estará dirigida por los valores de ICP / CPP.

Sin embargo, estudios recientes han cuestionado el límite inferior para definir la hipotensión en pacientes con lesión en la cabeza aislada. En un estudio de 15,733 pacientes con lesión en la cabeza de moderada a grave, se calcula el valor de la presión arterial sistólica de 110 mmHg o menos como el mejor modelo para la hipotensión cuando la mortalidad se tomó como medida de resultado(Berry, y otros, 2012). En otro estudio, se encontró que mantener una presión arterial sistólica de aproximadamente 120 mmHg es más eficaz para minimizar la lesión cerebral secundaria. Por lo tanto, se necesitan más ensayos clínicos grandes para respaldar estos hallazgos. Se recomienda que los pacientes con LCT grave tengan líneas arteriales y venosas centrales colocadas tan pronto como sea posible. 


\section{Atención prehospitalaria en trauma craneoencefálico}

Vol. 3, núm. 3., (2019)

Javier Fabricio Valdez Zambrano; Alex William Marín Cherrez; Verónica Alexandra Muñoz Avilés; Joselyn Anabell Minda Reyes

La hipertensión puede ser una respuesta fisiológica producida debido a una oleada simpática, y puede significar una perfusión cerebral comprometida. La hipertensión no debe tratarse a menos que se haya excluido o tratado una causa y la presión arterial sistólica sea >180$200 \mathrm{mmHg}$ o el MAP sea >110-120 mmHg. Disminuir el aumento de la presión arterial, como mecanismo compensatorio para mantener una CPP adecuada, exacerba la isquemia cerebral. Para el tratamiento de la hipertensión para alcanzar los objetivos de CPP, una infusión de betabloqueantes de acción corta es muy útil, lo que mantienepresión sanguínea. Estos agentes no causan vasodilatación cerebral en comparación con los nitratos y los bloqueadores de los canales de calcio y, por lo tanto, no aumentan el volumen sanguíneo cerebral y la PIC.

Resucitación fluida.

Además de esto, la pronta reanimación con fluidos es de suma importancia para preservar la CPP óptima. Sin embargo, la elección de fluidos sigue siendo una cuestión de gran conflicto. Algunos estudios han sugerido que los líquidos hipertónicos podrían suprimir los biomarcadores de LCT y se correlacionan con un mejor resultado. Muchos estudios han investigado el efecto de varios fluidos en la reanimación prehospitalaria. Ningún estudio ha informado una mejor supervivencia y resultados funcionales sobre el uso de cristaloides isotónicos.

PH.

La hipotermia tiene algunos efectos protectores en la LCT al reducir la PIC, las demandas metabólicas cerebrales, disminuir la interrupción de la barrera hematoencefálica e inhibir la cascada inflamatoria. La hipotermia inducida es ahora una medida aceptada para mejorar el 


\section{Atención prehospitalaria en trauma craneoencefálico}

Vol. 3, núm. 3., (2019)

Javier Fabricio Valdez Zambrano; Alex William Marín Cherrez; Verónica Alexandra Muñoz Avilés; Joselyn Anabell Minda Reyes

resultado después de una lesión cerebral anóxica asociada con un paro cardíaco, pero sus beneficios en la LCT no son concluyentes en la actualidad.

\section{Conclusión.}

Los pacientes graves con LCT imponen una mortalidad y morbilidad muy altas y, sorprendentemente, una parte sustancial de estos malos resultados puede prevenirse mediante una evaluación rápida y un tratamiento prehospitalario, así como el tratamiento de emergencia temprano de tales casos. Según la evidencia, la mayoría de las estrategias de manejo comprenden la corrección rápida de la hipoxemia y la hipotensión, los dos predictores más importantes de mortalidad. Sin embargo, todavía existe la necesidad de definir los objetivos para el manejo de la hipotensión y la inclusión de nuevos carros de vía aérea difíciles, así como dispositivos de monitoreo adecuados para garantizar una mejor intubación y manejo ventilatorio. La solución salina isotónica debe usarse como la primera opción para la reanimación con líquidos. El PH tiene más efectos adversos y, por lo tanto, debe evitarse.

La mayoría de los ensayos de gestión publicados se han centrado principalmente en el tratamiento, así como en las estrategias de prevención de la lesión cerebral secundaria. Los resultados de estos ensayos ciertamente serían adoptados por nuevas pautas estandarizadas y, por lo tanto, pueden tenerun impacto sustancial en el manejo prehospitalario en pacientes con LCT.

\section{Bibliografia.}

Badjatia, N., Carney, N., Crocco, T., Fallat, M., Hennes, H., \& Jagoda, A. (2008). Brain Trauma Foundation; BTF Center for Guidelines Management. Prehosp Emerg Care , 51-52. 


\section{Atención prehospitalaria en trauma craneoencefálico}

Vol. 3, núm. 3., (2019)

Javier Fabricio Valdez Zambrano; Alex William Marín Cherrez; Verónica Alexandra Muñoz Avilés; Joselyn Anabell Minda Reyes

Berlot, G., La Fata, C., Bacer, B., Biancardi, B., Viviani, M., \& Lucangelo, U. (2009). Infl uence of prehospital treatment on the outcome of patients with severe blunt traumatic brain injury: A single-centre study. Eur J Emerg Med, 312-317.

Bernard, S., Nguyen, V., Cameron, P., Masci, K., Fitzgerald, M., \& Cooper, D. (2010). Prehospital rapid sequence intubation improves functional outcome for patients with severe traumatic brain injury: A randomized controlled trial. Ann Surg, 959-965.

Berry, C., Ley, E., Bukur, M., Malinoski, D., Margulies, D., Mirocha, \& J. (2012). Redefining hypotension in traumatic brain injury. Injury, 1833-1837.

Bolívar, J. (2015). Investigación Documental. México. Pax.

Bulger, E., Guffey, D., Guyette, F., MacDonald, R., Brasel, K., \& Kerby, J. (2012). Impact of prehospital mode of transport after severe injury: A multicenter evaluation from the Resuscitation Outcomes Consortium. J Trauma Acute Care Surg, 567-73.

Bullock, M., \& Povlishock, J. (2007). Brain Trauma Foundation, American Association of Neurological Surgeons, Congress of Neurological Surgeons, AANS/CNS Joint Section on Neurotrauma and Critical Care. Guidelines for the management of severe traumatic brain injure. J Neurotauma, 106.

Castro, J. (2016). Técnicas Documentales. México. Limusa.

Caterino, J., \& Raubenolt, A. (2012). The prehospital simplifi ed motor score is as accurate as the prehospital Glasgow coma scale: Analysis of a statewide trauma registry. Emerg Med J, 492-496.

Chi, J., Knudson, M., Vassar, M., McCarthy, M., Shapiro, M., \& Mallet, S. (2006). Prehospital hypoxia affects outcome in patients with traumatic brain injury: A prospective multicenter study. J Trauma, 1134-1141.

Davila, A. (2015). Concepto de terminos cientificos. Caracas: Oasis.

Myburgh, J., Cooper, D., Finfer, S., Venkatesh, B., Jones, D., \& Higgins, A. (2008). Australasian Traumatic Brain Injury Study (ATBIS) Investigators for the Australian; f, New Zealand Intensive Care Society Clinical Trials Group. Epidemiology and 12-month outcomes Australasian Traumatic Brain Injury Study (ATBIS) Investigators for the Aus. J Trauma, 864-870.

Pearson, W., Ovalle, F., Faul, M., Sasser, \& SM. (2012). A review of traumatic brain injury trauma center visits meeting physiologic criteria from The American College of Surgeons Committee on Trauma/Centers for Disease Control and Prevention Field Triage Guideline. Prehosp Emerg Care, 323. 


\section{Atención prehospitalaria en trauma craneoencefálico}

Vol. 3, núm. 3., (2019)

Javier Fabricio Valdez Zambrano; Alex William Marín Cherrez; Verónica Alexandra Muñoz Avilés; Joselyn Anabell Minda Reyes

Wu, X., Hu, J., Zhuo, L., Fu, C., Hui, G., \& Wang, Y. (2008). Epidemiology of traumatic brain injury in eastern China, 2004: A prospective large case study. J Trauma, 1313-1319. 